\title{
Electrohydrodynamic-Driven Micromixing for the Synthesis of Highly Monodisperse Nanoscale
}

\section{Liposomes}

Paresa Modarres* and Maryam Tabrizian †

*†Department of Biomedical Engineering, McGill University, Montreal, Canada

* paresa.modarres@ mail.mcgill.ca

$\dagger$ maryam.tabrizian@mcgill.ca 


\section{DEVICE GEOMETRY}

The electrode and microfluidic channel geometrical parameters are provided in Figure S1 and Table S1.

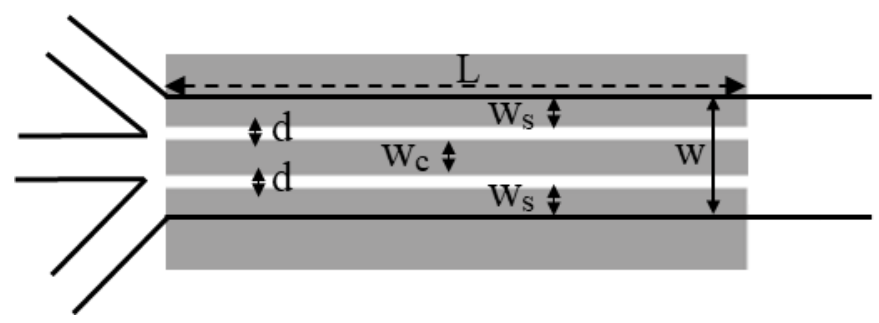

Figure S1. Geometrical parameters of the electrohydrodynamic micromixer.

Table S1. Geometrical parameters corresponding to Figure S1.

\begin{tabular}{ccc}
\hline Parameter & Value $(\boldsymbol{\mu m})$ & Description \\
\hline w $_{\mathrm{c}}$ & 60 & Center electrode \\
$\mathrm{w}_{\mathrm{s}}$ & 90 & Side electrode \\
$\mathrm{d}$ & 20 & Electrode spacing \\
$\mathrm{w}$ & 280 & Channel width \\
$\mathrm{h}$ & 60 & Channel height \\
$\mathrm{L}$ & 3000 & Electrode length \\
\hline
\end{tabular}

\section{DEVICE FABRICATION: PDMS OLIGOMER EXTRACTION}

It is well known that un-cross-linked PDMS oligomers in bulk polymerized PDMS can leach in organic solvents producing a source of unaccounted contamination in PDMS-based microdevices. ${ }^{1}$ To eliminate the un-cross-linked oligomers, an extraction protocol was employed that involved submersion of PDMS pieces in ethyl acetate (5 days) and acetone (2 days) followed by drying at $90{ }^{\circ} \mathrm{C}$ in a convection oven for 1 day. The degree of PDMS oligomer release was quantified for 
the raw and extracted PDMS using 4 common solvents including acetone (Sol1), 2-propanol (Sol2), ethanol (Sol3), and acetonitrile (Sol4). The raw and extracted PDMS pieces $(2 \mathrm{~cm} \times 1 \mathrm{~cm}$ $\times 0.3 \mathrm{~cm}$ blocks) were weighed and then immersed in $5 \mathrm{~mL}$ of each solvent for 6 hours with no agitation at room temperature. Then, the pieces were removed from the solvents and dried in a 90 ${ }^{\circ} \mathrm{C}$ oven for 1 day. The weight of untreated and extracted PDMS pieces was measured after solvent evaporation and the weight change percentage is reported in Figure S2. The weight reduction was a result of oligomer removal from the PDMS matrix. As illustrated in Figure S2, the extraction protocol employed in this study was very effective in eliminating unpolymerized oligomers. For instance, the $2 \%$ weight reduction for untreated PDMS was lowered to $0.04 \%$ for the extracted PDMS in acetone. A negligible weight change was observed for ethanol that is less aggressive compared to acetone. ${ }^{1}$

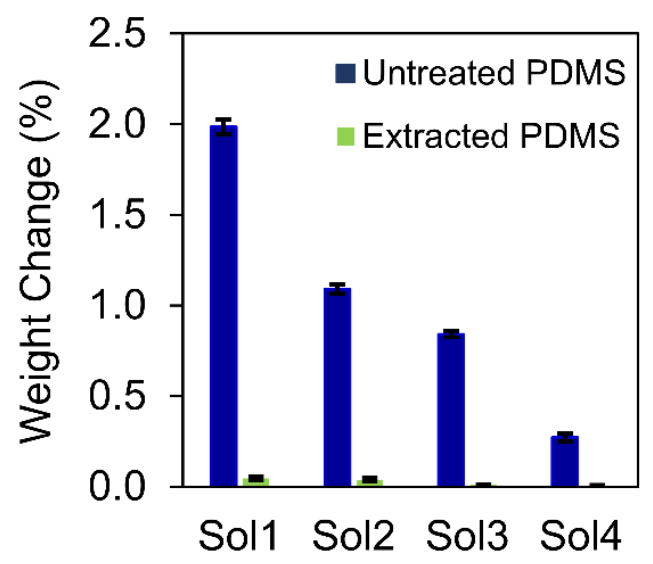

Figure S2. Weight percentage change of untreated and extracted PDMS blocks before and after submersion in solvents. Sol1: acetone, Sol2: 2-propanol, Sol3: ethanol, Sol4: acetonitrile.

\section{CONTROL EXPERIMENTS}

To ensure the liposomal batches were free from contaminates, control experiments were carried out to rule out the presence of any nanoparticle contaminates either due to the ethanol impurities or the metal-generated nanoparticles. ${ }^{2-3}$ The control experiments were run by flowing different ratios of DI water to ethanol and measuring the output by the NTA. For comparison, the NTA 
image snapshots for the liposomal batches of $0.2 \mathrm{mg} / \mathrm{mL}$ lipid concentration were contrasted with those of control experiments (Figure S3). In this figure, four randomly selected frames from NTA videos corresponding to each experimental condition are shown. As indicated by Figure S3 and the average number of particles per frame reported by the NTA, the control experiments showed a negligible amount of contaminates.

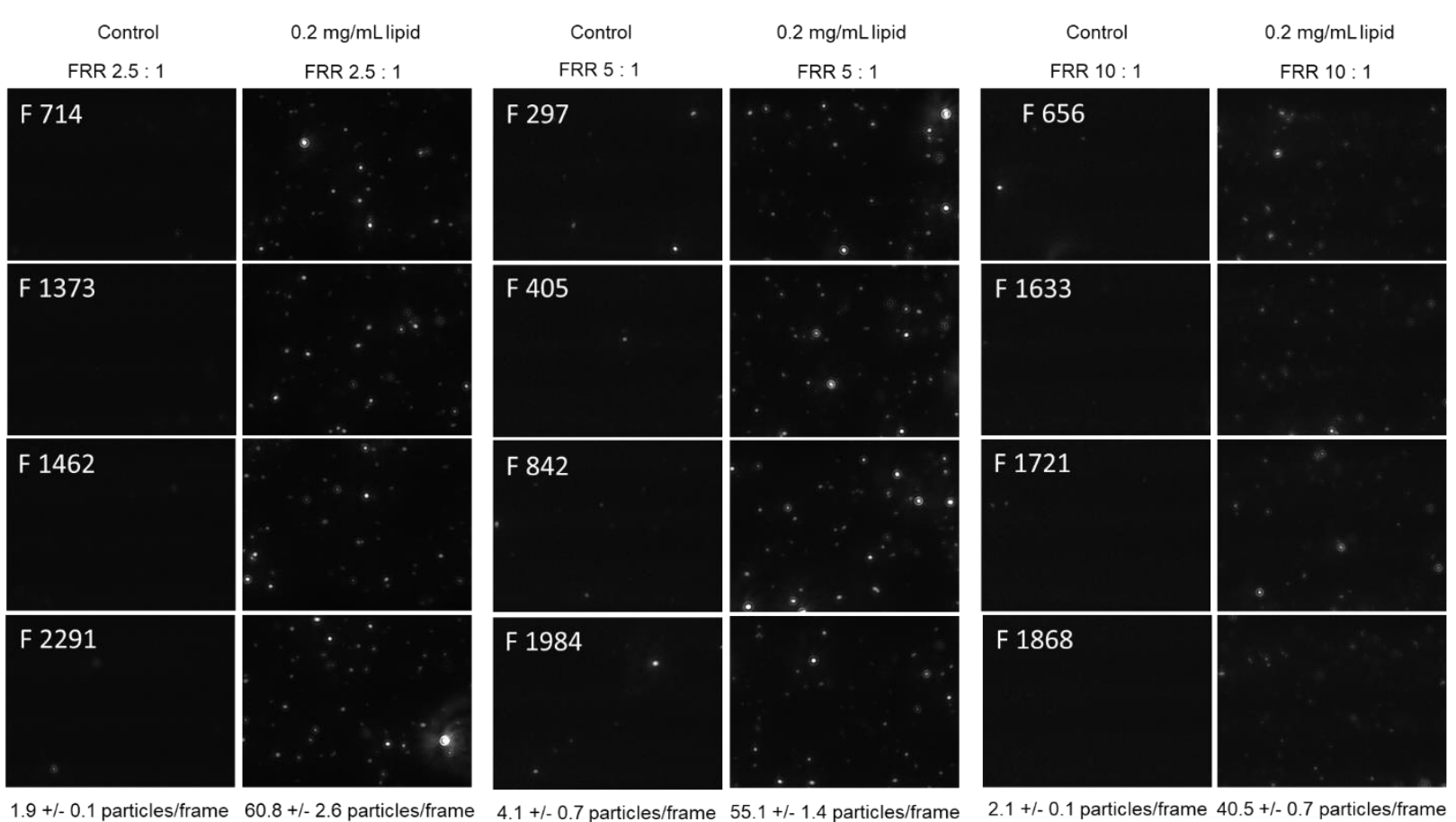

Figure S3. Control experiments for different FRR of DI water to ethanol solution without any dissolved precursors. For each experimental condition, four randomly chosen frames were selected from the recorded NTA videos. The number on the left top corner of each image corresponds to its frame number.

\section{REFERENCES}

1. Lee, J. N.; Park, C.; Whitesides, G. M., Solvent compatibility of poly(dimethylsiloxane)-based microfluidic devices. Analytical Chemistry 2003, 75 (23), 6544-6554.

2. Alheshibri, M.; Craig, V. S. J., Generation of nanoparticles upon mixing ethanol and water; Nanobubbles or Not? Journal of Colloid and Interface Science 2019, 542, 136-143.

3. Rak, D.; Ovadova, M.; Sedlak, M., (Non)Existence of Bulk Nanobubbles: The Role of Ultrasonic Cavitation and Organic Solutes in Water. J Phys Chem Lett 2019, 10 (15), 42154221. 